\title{
Analysis of dual clock detection probability Random Multiple Access Protocol
}

\author{
Zhi Xul,a ${ }^{1, \text { }}$ Yifan Zhao ${ }^{1}$, Hongwei Ding ${ }^{1}$, Shengjie Zhou ${ }^{1}$,Longjun Liu $^{1}$ \\ ${ }^{1}$ School of Information, Yunnan University, Kunming 653000, China. \\ a775862901@qq.com
}

Keywords: idle period, CSMA, throughput.

\begin{abstract}
In wireless sensor networks, traffic load moment node are changing ;the basis of CSMA protocol for idle channels not do effectively. To address this problem, analysis the lack of basic CSMA protocol, in order to improve the utilization of CSMA protocol idle, we come up with a new protocol which is the double clock P detection CSMA random multiple access protocol. Using the average cycle[1] approach to protocol analysis, and finally got the mathematical expression of system throughput. Final simulation results show the advantage of dual clock random probability of detection CSMA multiple access protocol , make full use of channel's idle, and this agreement can remain relatively stable throughput at a high load.
\end{abstract}

\section{Introduction}

With the development of information exchange, data transmission and volume of interactive into an explosive growth, we have reached the era of big data [2], everything should use the data to manage. The automobile industry as a pillar industry in recent years, the continuous development of the internal, the car has become an indispensable daily general merchandise. In the interconnection of all things the most distinctive is the car networking[3]. But in this development process, the data transmission inefficiencies remain binding on the development of multi-vehicle networking system for data exchange. In order to improve the efficiency of data transmissions, so the wireless sensor network[4] might be into deeper study. There are many nodes in a wireless sensor network, but the channel resources are limited. If the node does not regularly send information, it will lead to an information packet collision occurs, the collision rate of the system will be greatly increased. But if we use the CSMA (Carrier Sense Multiple Access) protocol, the channel is being observed, if the channel is idle, then the information packet will be sent; if the channel is busy, then delay sending information packets. This will reduce the collision rate, improve channel efficiency.

Fundamental CSMA protocols are: non-persistent CSMA protocol, 1-persistent CSMA protocol, P-persistent CSMA protocol, as well as the probability of detection CSMA protocol. In these four protocols, the probability of detection CSMA protocol performance best. These agreements all have their own characteristics, but there are also some defects, have failed to make full use of idle period. Therefore, in order to improve system throughput. This article introduces a dual clock probability of detecting a random multiple access protocol to obtain a theoretical formula by mathematical analysis, and simulation probability of detecting a double clock and other CSMA agreements for comparison, the probability of detecting a dual clock Random Multiple Access Protocol have better throughput, Improve the efficiency of data transmission at the same time.

\section{Dual clock detection probability CSMA model}

In dual clock probability of detecting a random multiple access protocol, if there is a system which is sending the Information packet, the timeline of system will be divided into time slots[5]. The maximum transmission delay of the system is a, set it to the slot length , and then set packet length per unit length, which is an integer multiple of a .Three random events, the $U$ event(successful events) and B event(collision event)merged BU event(busy event)

In this model, there will be three random events, namely:

(1)Event that information packets are sent successfully (U events); 
(2)Event that there are no information packets in the channel, the channel is idle (I event);

(3)Event that information packets collide with each other (collision event , B event).

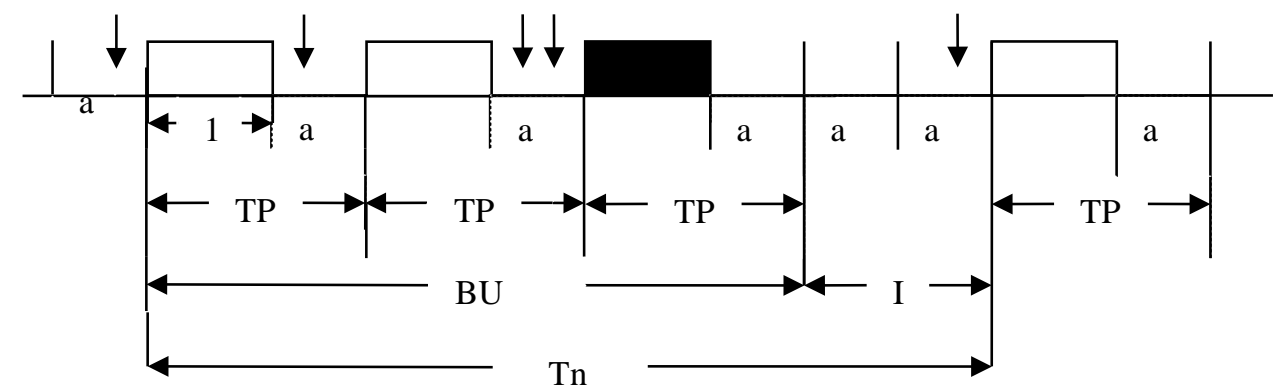

Fig1. The model of Dual clock detection probability CSMA

In Figure 1, when the system is no packet arrives, that is idle, the system axis is continuous. Information packets arrive in busy period ,the channel will be continued to be supervised. when the channel is detected idle, in the beginning of the next slot with probability $\mathrm{P}$ is sent with probability 1-P abandoning transmission; when the information packet arrives in the idle period, which is continuous clock, the same with probability $\mathrm{P}$ is sent, and probability 1-P abandoning transmission. Because this system combines the advantages of continuous and discrete systems. So that system performance is improved.

\section{Throughput of the new protocol}

Before system performance analysis, to make the following assumptions: the channel is ideal with no noise and interference; The basic unit of the system control clock is a, the information packets arrived at time a will transmit at starting time of the next slot; The channel propagation delay is a, the packet length is unit length and is an integral multiple of a.

During the transmission of information packets, the phenomenon of pocket collisions occur inevitably, and continues to be sent after a random time delay, it sends will not produce any adverse effects on the arrival process channel.

Variable makes the following assumptions:

(a) U represents a successful packet transmission events, B represents a packet collision incident , I represents an idle event, BU represents successful joint event with the collision;

(b) $P\left\{N_{B U=i}\right\}$ represents a probability that the i BU consecutive event occur within the busy period; $P\left\{N_{I}=j\right\}$ represents the probability of a cycle of event $\mathrm{I} \mathbf{j}-$ th consecutive; $P\left\{N_{I}=j, N_{B U}=i\right\}$ represents a joint probability of the $\mathrm{j}-$ th cycle and the $\mathrm{i}-\mathrm{th}$ event $\mathrm{I}$ and BU consecutive events; $S$ represents throughput ; Information packet arrival probability satisfy Poisson distribution: $\mathrm{P}(\mathrm{n})=\frac{(\mathrm{tG})^{\mathrm{n}} \mathrm{e}^{-\mathrm{tG}}}{\mathrm{n} !}$; The probability of a channel slot with no information packet arrival is $\mathrm{e}^{-\mathrm{aG}}$; The probability of no channel information packet arrival in transmission period is: $\mathrm{e}^{-\mathrm{P}(1+\mathrm{a}) \mathrm{G}}$.According to the obtained, it can be analyzed to obtain the probability of the following events : First, obtain the probability of continuous events $P\left(N_{B U}=i\right)=(1-$ $\left.\mathrm{e}^{-\mathrm{aG}}\right)\left(1-\mathrm{e}^{-\mathrm{P}(1+\mathrm{a}) \mathrm{G}}\right)^{\mathrm{i}-1} \quad ;$ Next, obtain the probability of continuous events $P\left(N_{I}=j\right)=e^{-P(1+a) G}\left(e^{-a G}\right)^{j-1}$; Finally, the joint probability of the event BU and event I distribution function:

$$
P(i, j)=\left(1-e^{-a G}\right)\left(1-e^{-P(1+a) G}\right)^{i+1} e^{-P(1+a) G}\left(e^{-a G}\right)^{j-1}
$$

First we should solve the average length of event $\mathrm{E}(\mathrm{U})$. In the new protocol, the information packet can be sent successfully divided into the following two situations:

Only an information packet arrives in the last slot of idle period, then the information packet is sent in the next time slot, the event is $U_{1}$, the average length of $U_{1}$ : 


$$
\mathrm{E}\left(\mathrm{U}_{1}\right)=\mathrm{E}\left(\mathrm{i}_{\mathrm{U}_{1}}\right) \times 1=\frac{\mathrm{aGe}}{1-\mathrm{e}^{-\mathrm{aG}}}
$$

information packets in the busy period (within TP), this information and the current packet is a unique period TP probability $\mathrm{P}$ listener to an information packet, the information packet is successfully transmitted within a next slot. This event is $U_{2}$ :

$\mathrm{E}\left(\mathrm{U}_{2}\right)=\sum_{\mathrm{i}=1}^{\infty} \sum_{\mathrm{j}=1}^{\infty} \sum_{\mathrm{k}=0}^{\mathrm{i}-1} \mathrm{kP}(\mathrm{i}, \mathrm{j})=\mathrm{P}(1+\mathrm{a}) \mathrm{G}$

$\mathrm{E}(\mathrm{U})=\mathrm{E}\left(\mathrm{U}_{1}\right)+\mathrm{E}\left(\mathrm{U}_{2}\right)=\frac{\mathrm{aGe} \mathrm{e}^{-\mathrm{aG}}}{1-\mathrm{e}^{-\mathrm{aG}}}+\mathrm{P}(1+\mathrm{a}) \mathrm{G}$

Then we should solve the average length of the busy event $E(B U)=\frac{1+a}{e^{-P(1+a) G}}$; Finally, solving the average length of idle time I, Since the number of slots with mean $E[N]=\frac{1}{1-e^{-a G}}$ geometric distribution during the idle period I, So the normalized probability of information packets reaches in a time slot: $\mathrm{P}_{1}=\frac{\mathrm{aGe}}{1-\mathrm{e}^{-\mathrm{aG}}}$; the normalized probability of more than one message packets reaches in time slot: $\mathrm{P}_{2}=\frac{1-\mathrm{aGe}^{-\mathrm{aG}}-\mathrm{e}^{-\mathrm{aG}}}{1-\mathrm{e}^{-\mathrm{aG}}}$; Then there are:

$\mathrm{E}(\mathrm{I})=\left(\frac{1}{1-\mathrm{e}^{-\mathrm{aG}}}-1\right) \times \mathrm{a}+\frac{\mathrm{aGe}^{-\mathrm{aG}}}{1-\mathrm{e}^{-\mathrm{aG}}} \times \frac{\mathrm{a}}{2}+\frac{1-\mathrm{aGe}^{-\mathrm{aG}}-\mathrm{e}^{-\mathrm{aG}}}{1-\mathrm{e}^{-\mathrm{aG}}} \times \frac{\mathrm{a}}{2}$

Therefore, according to the above requirements can be obtained throughput.

\section{Dual clock detection probability CSMA Simulation and Analysis}

Based on the above analysis, the use of simulation software Matlab R2012b dual clock probability of detecting a communication system Random Multiple Access Protocol under simulated. The whole simulation process, delay $\mathrm{a}=0.1$, the information packet length is 1 .

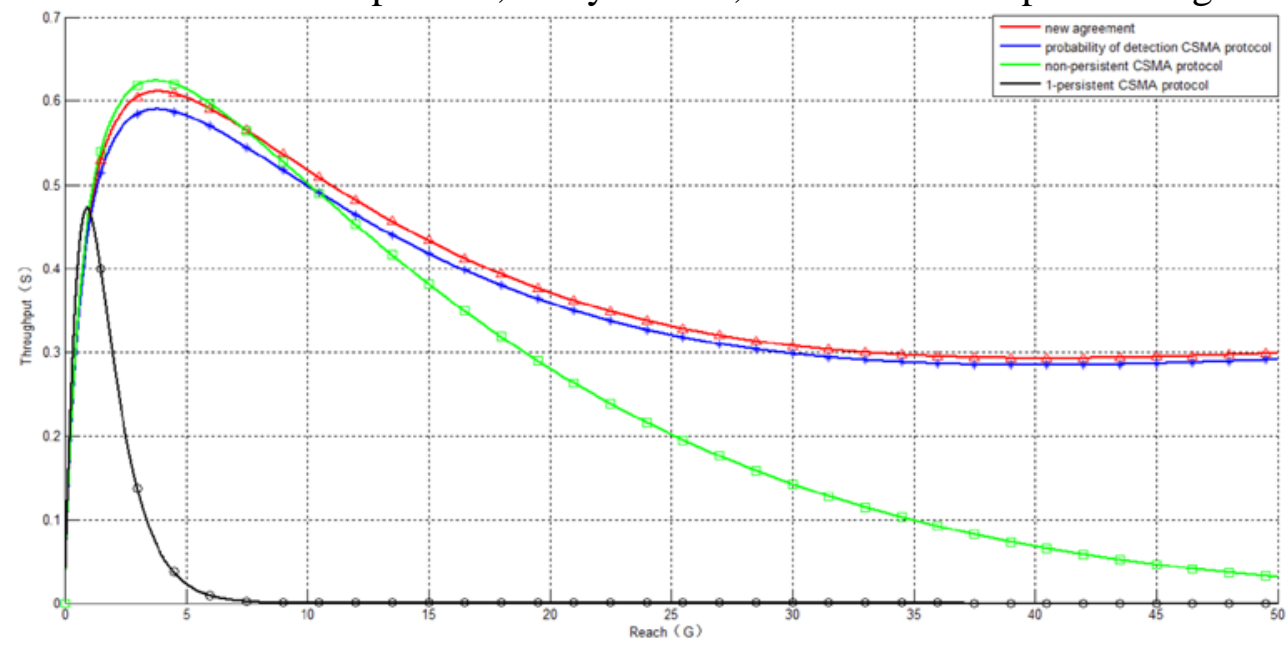

Fig2. Compare the throughput of the new protocol and the other three basic protocols

As shown in Figure 2, the new protocol and CSMA protocol probability of detecting non-persistent CSMA protocol, and 1-persistent CSMA protocol throughput was clear contrast; The probability of detection CSMA protocol has shown a steady overall advantage, and the dual clock probability of detection CSMA on this basis to enhance the system throughput in further. And it improves the transmission efficiency of the system in some aspects.

(a) Non-persistent CSMA protocol throughput is the highest, but the utilization of channel is very low, when the arrival rate increases, the system throughput decreases very fast, showing its shortage.

(b) 1-persistent CSMA protocol throughput compared with the other three protocols, the throughput is the lowest, and when there is more load, will produce more collision, the throughput will soon be reduced to zero, the system performance lower.

(c) The probability of detection CSMA protocol combines the above-mentioned agreement, Although slightly lower than the throughput of non-persistent CSMA protocol in the early time, the arrival rate increases throughput and slow decline, to enhance the stability of the system throughput. 
(d) New agreement(dual clock probability of detecting CSMA protocol) on the basis of probability of detection CSMA protocol, the system idle period has been adjusted, combines the advantages of continuous and discrete systems, thereby reducing the idle rate increase throughput, finally showing the advantages of the new agreement, to ensure system stability and improve system throughput.

\section{Summary}

In this paper, based the probability of detection CSMA, the proposed dual clock probability of detecting CSMA protocol, after the adoption of the new agreement, the system can detect CSMA protocol based on the increase in the probability of higher throughput and guaranteed throughput in heavy load under still able to maintain high system throughput and enhance the performance of wireless communication system. Meanwhile, the simulation results demonstrate the consistency of simulation experiments and theoretical analysis value by comparing different throughput of CSMA protocol, confirming the dual clock probability of detecting CSMA protocol advantages over other CSMA protocol in the case of load change.

\section{Acknowledgements}

This work was supported by the National Natural Science Foundation of China (61461053, 61461054, 61072079); Natural Science Foundation of Yunnan Province (2010CD023); The Financial Support of Yunnan University (No.XT412004).

\section{References}

[1] Dongfeng Zhao. Analysis study on the method of S - ALOHA system average period. Journal of Yunnan university, 1994, 16 (2) : 133-138.

[2] Yihua Du, big data under the background of the Chinese academy of sciences scientific research management data center construction $[\mathrm{J}]$ _ computer system application are discussed. 2015 (1) : 79-87

[3] China mobile communications group Jiangsu co., LTD. Car networking development must cross-border fusion [z]. 2015

[4] Dongfeng Zhao. The random reservation time division multiple access wireless communication network, the 1987 national conference on graduate academic communications, [C]. 1987,10:93-98.

[5] Dongfeng Zhao. Time slot type multiple access system analysis method research [J]. Journal of university of electronic science and technology, 1998, 27 (6): 588-591. 